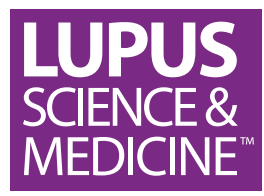

\title{
Prevalence of systemic lupus erythematosus-related symptoms assessed by using the Connective Tissue Disease Screening Questionnaire in a large population-based cohort
}

\author{
Wietske Lambers (D) , ${ }^{1}$ Suzanne Arends, ${ }^{1}$ Caroline Roozendaal, ${ }^{2}$ \\ Elisabeth Brouwer, ${ }^{1}$ Hendrika Bootsma, ${ }^{1}$ Johanna Westra,${ }^{1}$ Karina de Leeuw ${ }^{1}$
}

To cite: Lambers W, Arends S, Roozendaal C, et al. Prevalence of systemic lupus erythematosus-related symptoms assessed by using the Connective Tissue Disease Screening Questionnaire in a large population-based cohort. Lupus Science \& Medicine 2021;8:e000555. doi:10.1136/ lupus-2021-000555

- Additional supplemental material is published online only. To view, please visit the journal online (http://dx.doi.org/10. 1136/lupus-2021-000555).

Received 25 August 2021 Accepted 27 October 2021

Check for updates

(c) Author(s) (or their employer(s)) 2021. Re-use permitted under CC BY-NC. No commercial re-use. See rights and permissions. Published by BMJ.

${ }^{1}$ Clinical Immunology and Rheumatology, UMCG, Groningen, The Netherlands

${ }^{2}$ Department of Laboratory

Medicine, UMCG, Groningen, The Netherlands

Correspondence to Dr Wietske Lambers; w.m. lambers@umcg.nl

\section{ABSTRACT}

Background To assess the prevalence of self-reported SLE-related symptoms associated with demographic and biochemical data and connective tissue disease (CTD)related autoantibodies in a large population-based cohort. Methods Participants of the Dutch Lifelines population cohort filled out the Connective Tissue Disease Screening Questionnaire (CSQ), including 11 questions focusing on SLE-related symptoms (SLE-CSQ) based on the American College of Rheumatology classification criteria. CTD autoantibody screen was performed in $25 \%$ of participants.

Results Of 85295 participants with complete SLE-CSQ data, after excluding patients with SLE and other CTDs $(n=126), 41781$ (49.1\%) had no positively answered questions and 2210 ( $2.6 \%$ of total) had $\geq 4$ positive answers. Participants with $\geq 4$ answers on the SLE-CSQ were significantly younger, more frequently female, had lower body mass index (BMI) and were more often smokers than those with negative scores. Furthermore, counts of leucocytes, neutrophils and monocytes were significantly higher in these participants, while the levels of haemoglobin and creatinine were lower. CTD autoantibodies were present in $2.2 \%$ of participants with SLE-CSQ score of 0 , compared with $3.5 \%$ with SLECSQ score $\geq 4$ ( $p=0.001)$. Multivariate analysis showed, after adjusting for age, gender, BMl and smoking, that haemoglobin levels remained significantly lower in participants with SLE-CSQ score $\geq 4$.

Conclusions In this large population-based cohort, $2.6 \%$ of participants without diagnosed CTD reported $\geq 4$ positive answers on the SLE-CSQ, indicating high suspicion for SLE. These individuals had demographic and haematological characteristics that differed from the remaining population. Potentially, this questionnaire, in combination with autoantibody determination, can be used as a starting point of a screening cascade in order to detect SLE at an early stage.

\section{INTRODUCTION}

SLE is a systemic autoimmune disease characterised by the presence of ANA and

\section{Key messages}

What is already known about this subject?

- The presenting symptoms of SLE are non-specific and diverse, hindering early recognition and resulting in frequent diagnostic delay.

What does this study add?

- This study assesses the prevalence of self-reported SLE-related symptoms associated with demographic and biochemical data and connective tissue disease (CTD)-related autoantibodies in a large populationbased cohort.

- The study shows that $2.6 \%$ of the general population have SLE suspicion based on the questionnaire.

- CTD autoantibodies were significantly more often detectable in patients with high SLE suspicion.

How might this impact on clinical practice or future developments?

- Potentially, a screening questionnaire for SLE-related symptoms, in combination with autoantibody determination, can be used as a starting point of a screening cascade in order to detect SLE at an early stage.

multiorgan involvement. Prevalence rates range from 20 to 70 per $100000 .^{1}$ Women are more frequently affected than men, with an approximate ratio of 9:1.

The presenting symptoms of the disease are non-specific and diverse, hindering early recognition and resulting in frequent diagnostic delay. ${ }^{23}$ It has been shown that many patients with SLE had visited the general practitioner prior to diagnosis with fatigue, arthralgia, arthritis, rash, alopecia, sicca symptoms, Raynaud's phenomenon and/ or serositis. ${ }^{2} 3$ Besides clinical symptoms, immunological changes take place before the disease is uncovered, as autoantibodies can be detected in serum many years before diagnosis. $^{45}$ Hence, there is a window of 
opportunity for earlier diagnosis of SLE. Early identification of patients with SLE would allow timely diagnosis and treatment, probably preventing organ damage. ${ }^{67}$

The classification of SLE is historically based on the American College of Rheumatology (ACR) classification criteria, which were first published in 1982 and revised in 1997. ${ }^{89}$ A cumulative scoring system classifies SLE when 4 or more of 11 clinical and immunological symptoms are present. More recently, new classification criteria have been developed. First the 2012 Systemic Lupus International Collaborating Clinics criteria were published and later the 2019 European League Against Rheumatism (EULAR) /ACR classification criteria. ${ }^{1011}$

The Connective Tissue Disease Screening Questionnaire (CSQ) was developed to screen for various connective tissue diseases (CTD) in population studies. ${ }^{12}$ The questions that are included to detect SLE (SLE-CSQ) showed $96 \%$ sensitivity and $86 \%$ specificity for SLE in patients who were referred to a rheumatologist in the original study. Interestingly, in a population with firstdegree relatives of patients with SLE, the ones who transitioned to classified SLE had significantly higher SLE-CSQ scores than the remaining relatives. ${ }^{13}$ Therefore, the SLECSQ might contribute to early recognition of SLE.

To date, it is not clear what the potential use could be of a SLE-specific screening questionnaire in the general population. Also, it is not clear whether increased SLE suspicion based on such questionnaire will detect persons with specific demographic and biochemical characteristics similar to SLE, such as female predominance, mostly of reproductive age, and haematological features such as anaemia and leucopenia, as well as the presence of specific CTD autoantibodies.

In this study, the aim was to investigate the prevalence of SLE-related symptoms, assessed with the SLE-CSQ, and relate the outcome to demographic and biochemical data, and the presence of CTD autoantibodies, in a large population-based cohort.

\section{MATERIALS AND METHODS}

\section{Participants and data collection}

Data were obtained from the Lifelines cohort, which is a large population-based cohort among inhabitants of the northern region of the Netherlands. Inclusion of this prospective multidisciplinary cohort started in 2006, and 167729 persons participated. At baseline, participants were asked to fill out questionnaires about their health, nutritional behaviour, medical history and use of medication. Also, they underwent general clinical examination and blood withdrawal for general laboratory testing. All participants included in the current study were $\geq 18$ years old. In a follow-up questionnaire taken approximately 2 years after inclusion, participants were asked to fill out questions that were derived from a validated CSQ. ${ }^{12}$ Only participants who completed this follow-up questionnaire were included in the current study.
All participants provided written informed consent. Lifelines is open for all researchers and information is available at http:/ /wwwLifeLinesnl.

\section{Detection of patients with established SLE and other CTDs}

Participants who had already been diagnosed with SLE or with another CTD, namely primary Sjögren's syndrome (pSS), systemic sclerosis (SSc), mixed connective tissue disease (MCTD), and polymyositis or dermatomyositis (PM/DM), at baseline were excluded. These patients were detected using the lists of self-reported diseases. The diagnosis was confirmed by self-reported use of medication and whether participants reported to have visited a medical specialist in the past 12 months.

\section{Connective Tissue Disease Screening Questionnaire}

Collection of CSQ data ${ }^{12}$ took place after a median of 25 months (IQR 23-30 months) from baseline. In total, 11 questions were selected to compose an 'SLE-CSQ score' (see table 1). These questions are aiming for SLE-specific symptoms and are based on the 11 components of the ACR classification criteria, with a score $\geq 4$ suspicious for SLE. ${ }^{12}$ Arthritis was defined as joint swelling lasting more than 6 weeks in at least two joints. Raynaud's phenomenon was considered positive when at least two colour changes were reported to be shown on exposure to cold.

Sicca complaints of the eyes and mouth were separately documented in order to disclose any overlap between SLE and pSS.

\section{EliA CTD screen}

In a random selection of 21389 participants at baseline, EliA CTD screen (ThermoFisher Scientific, Freiburg, Germany) was performed in a blood sample on a Phadia 250 analyser, in which the total reactivity to a mixture of the following antigens is measured: human recombinant U1RNP (RNP70, A, C), SS-A/Ro (60 kDa, $52 \mathrm{kDa})$, SS-B/ La, centromere B, Scl-70, Jo-1, fibrillarin, RNA polymerase III, ribosomal P protein, PM-Scl, PCNA, Mi-2 proteins, Sm proteins and native purified DNA. The results are presented as a ratio, of which $>1.0$ was regarded positive, according to the manufacturer. Furthermore, in individuals with a positive CTD screen, levels of anti-dsDNA (double stranded DNA) and anti-SSA were measured using EliA on a Phadia 250 analyser. For both anti-dsDNA and anti-SSA, a cut-off of $>10 \mathrm{U} / \mathrm{mL}$ was considered positive, according to the manufacturer.

\section{Statistical analysis}

Descriptive results were expressed as percentage or median (IQR) for categorical and continuous data, respectively. $\chi^{2}$ test and Kruskal-Wallis test were used as appropriate to compare demographic, clinical and blood parameters between the groups based on SLECSQ score (score 0 , score 1 , score $2-3$, score $\geq 4$ ). In case this overall $\mathrm{p}$ value was statistically significant, $\chi^{2}$ test and Mann-Whitney $\mathrm{U}$ test were used to compare the variables between two groups. 
Table 1 SLE-CSQ and determination of the SLE-CSQ score ${ }^{12}$

1. Have you ever had swelling in any of the following joints, lasting more than 6 weeks?

A. Left wrist

B. Right wrist

C. Left finger joints (but not the joints nearest the fingernails)

D. Right finger joints (but not the joints nearest the fingernails)

E. Left elbow

F. Right elbow

G. Left knee

H. Right knee

2. Have your fingers ever shown any unusual colour changes in the cold?
A. If yes, was the colour white?
B. If yes, was the colour blue?
C. If yes, was the colour purple?
D. If yes, was the colour red?

3. Have you ever had sores in your mouth or nose for more than 2 weeks at a time? 1 point

4. Have you ever had a red rash on your cheeks for more than a month? 1 point

5. Have you ever had skin break out (rash) after being in the sun (not sunburn)? 1 point

6. Have you ever had pleurisy or chest pain made worse with deep breaths for 1 point more than a few days (not caused by bruised rib or a cold)

7. Have you ever had rapid loss of lots of hair?

1 point

8. A. Have you ever been told by a doctor that you had anaemia?

B. Have you ever been told by a doctor that you had low white cell count?

C. Have you ever been told by a doctor that you had low platelet count?

9. Have you ever been told by a doctor that you had protein in your urine? 1 point

1 point if any of these questions were positive

10. Have you ever had a blood test for lupus/SLE? (eg, ANA or ENA) If yes, what 1 point for positive result was the result?

11. Have you ever been told by a doctor that you had lupus/SLE of the skin? SLE-CSQ score

1 point

Cumulative score

ENA, extractable nuclear antibodies; SLE-CSQ, Connective Tissue Disease Screening Questionnaire focusing on SLE.

Multivariate multinomial logistic regression was performed to correct for possible confounders (age, gender, body mass index (BMI) and smoking status). Statistical analysis was performed using IBM SPSS Statistics V.23. $\mathrm{P} \leq 0.05$ was considered statistically significant.

\section{RESULTS}

\section{Study population}

The SLE-CSQ was completed by 85295 participants; 46 participants had SLE at the time of filling in the questionnaire and were excluded. This resulted in a prevalence of SLE of 54 per 100 000. Furthermore, 80 patients with other CTDs were excluded (46 pSS, 15 SSc, 5 MCTD, 14 PM/DM). Patients with SLE and other CTDs were significantly more often female, had lower haemoglobin level and lower lymphocyte count, and $66.7 \%$ of patients with SLE and other CTDs had detectable CTD autoantibodies.

The baseline characteristics of the 85169 included participants and the excluded patients with SLE and CTD are shown in table 2. The median age was 45 years and $59.6 \%$ were female. CTD autoantibody screen was assessed in $21359(25 \%)$ participants and was positive in $2.7 \%$.

\section{SLE-CSQ questionnaire}

The distribution of the number of positively answered SLE-CSQ questions among the population is shown in table 3. Almost half $(49.1 \%)$ of the participants had an SLE-CSQ score of 'zero' and thus reported no SLErelated symptoms. A score of at least four positive questions, raising increased suspicion for SLE, was found in $2.6 \%$ of the participants. In comparison, $34(73.9 \%)$ of (excluded) 46 patients with SLE and $27.8 \%$ of patients with other CTDs had at least four positive questions. The percentages of positively answered questions per CSQ item can be found in table 4 . The most frequently occurring symptoms in the population-based cohort were symptoms compatible with photosensitivity (24\%) and haematological features $(22 \%)$.

For further analysis, four subgroups were formed, namely groups with SLE-CSQ scores of $0,1,2$ or 3 and $\geq 4$. The characteristics and comparison of these subgroups are shown in table 5. Participants with SLE-CSQ score $\geq 4$ were younger (median age 43 years) than participants with a score of 0 and had a strong female predominance $(91 \%)$ compared with all groups with lower scores. Also, participants with the highest SLE-CSQ scores were more 
Table 2 Baseline characteristics of all participants

\begin{tabular}{|c|c|c|c|c|c|}
\hline Characteristics & $\begin{array}{l}\text { All (no SLE, no CTD) } \\
(\mathbf{n}=\mathbf{8 5 1 6 9 )}\end{array}$ & SLE $(n=46)$ & P value* & Other CTDs $(n=80)$ & $P$ value* \\
\hline Age (years) & $45(36-53)$ & $45(41-51)$ & 0.66 & $50(44-50)$ & $<0.0001$ \\
\hline Gender (female) & 59.6 & 93.5 & $<0.0001$ & 82.5 & $<0.0001$ \\
\hline BMI $\left(\mathrm{kg} / \mathrm{m}^{2}\right)$ & $25(23-28)$ & $25(22-28)$ & 0.44 & 25 (23-29) & 0.42 \\
\hline \multicolumn{6}{|l|}{ Smoking } \\
\hline Current (yes) & 18.4 & 23.9 & 0.36 & 13.8 & 0.26 \\
\hline Never (yes) & 47.2 & 55.6 & 0.53 & 45.0 & 0.76 \\
\hline \multicolumn{6}{|l|}{ Women only } \\
\hline Nulliparity (yes) & 18.4 & 17.0 & 0.68 & 8.6 & 0.06 \\
\hline Menopausal (yes) & 7.0 & 4.7 & 0.29 & 1.8 & 0.12 \\
\hline \multicolumn{6}{|l|}{ OCP use } \\
\hline Ever (yes) & 91.6 & 90.7 & 0.77 & 92.4 & 0.23 \\
\hline Current (yes) & 37.9 & 27.9 & 0.31 & 15.2 & 0.006 \\
\hline \multicolumn{6}{|l|}{ Blood levels } \\
\hline Haemoglobin (g/L) & $14.0(13.2-15.0)$ & $13.2(12.7-14.0)$ & $<0.0001$ & $13.4(12.6-13.9)$ & $<0.0001$ \\
\hline Leucocytes (×10\%/L) & $5.7(4.9-6.8)$ & $5.6(4.3-7.2)$ & 0.51 & $5.55(4.40-7.00)$ & 0.14 \\
\hline Lymphocytes (×10\%/L) & $1.9(1.6-2.3)$ & $1.72(1.41-2.22)$ & 0.03 & $1.58(1.19-2.10)$ & $<0.0001$ \\
\hline Neutrophils $\left(\times 10^{9} / \mathrm{L}\right)$ & $3.1(2.5-3.8)$ & $3.28(2.29-4.57)$ & 0.61 & 3.21 (2.34-3.92) & 0.70 \\
\hline Monocytes (×109/L) & $0.45(0.37-0.55)$ & $0.54(0.38-0.65)$ & 0.023 & $0.51(0.36-0.62)$ & 0.081 \\
\hline Thrombocytes $\left(\times 10^{9} / \mathrm{L}\right)$ & $245(212-283)$ & $260(233-204)$ & 0.051 & 256 (227-298) & 0.16 \\
\hline Creatinine $(\mu \mathrm{mol} / \mathrm{L})$ & $72(65-82)$ & $69(63-80)$ & 0.27 & $67(61-74)$ & $<0.0001$ \\
\hline $\begin{array}{l}\text { CTD screen positive } \\
(n=21359,25 \%)(\%)\end{array}$ & $580 / 21.359(2.7)$ & $8 / 12(66.7)$ & $<0.0001$ & $12 / 18(66.7)$ & $<0.0001$ \\
\hline $\begin{array}{l}\text { Anti-doublestranded } \\
\text { DNA" positive }(\% \dagger)\end{array}$ & $216 / 580(37)$ & $3 / 8$ (38) & & 1/12 (8) & \\
\hline Anti-SSA positive (\%†) & 152/580 (26) & $5 / 8(63)$ & & $5 / 12(42)$ & \\
\hline
\end{tabular}

Data are presented as median (IQR) for continuous variables and as percentages for categorical variables.

${ }^{*} P$ values represent statistical differences between patients with SLE and other CTDs, and the remaining participants, using Mann-Whitney $U$ test and $\chi^{2}$ test as appropriate.

†Percentage refers to the group of CTD screen-positive participants.

$\mathrm{BMI}$, body mass index; CTD, connective tissue disease; OCP, oral contraceptive pills.

frequently smokers than participants with no positive answers or with two to three positive answers. Among women, ever having used oral contraceptive pills (OCP) was reported more frequently, whereas current use of OCP was less frequent in the group with the highest SLECSQ scores. Furthermore, the percentage of participants with positive CTD autoantibody screen was higher in individuals with the highest SLE-CSQ scores, but only in comparison with the group with a score of 0 . There was no difference regarding percentage of anti-dsDNA antibodies between the groups, but anti-SSA seemed more prevalent in the group with a score $\geq 4$, although this was only statistically significant compared with score group 1 . Regarding the remaining laboratory findings, the levels of leucocytes and neutrophils (but not lymphocytes) as well as thrombocytes were higher in the group with high SLE-CSQ scores, while the levels of haemoglobin, creatinine and monocyte counts were lower compared with an SLE-CSQ score of 0 .
Independent predictors of SLE-CSQ score $\geq 4$

When adjusting for gender, age, BMI and smoking status in all participants, those with an SLE-CSQ score of $\geq 4$ had significantly lower haemoglobin levels than groups with scores of 0 and 1 , whereas neutrophil counts were higher compared with groups with lower scores, and the number of leucocytes was higher only than the group with scores of 2-3 (see table 6). For women, when adjusting for potential confounders (age, BMI and smoking status), those with SLE-CSQ score $\geq 4$ were more often nulliparous, less often menopausal, less often currently used OCP and more frequently had ever used OCP than participants who answered negatively to all SLE-CSQ questions.

\section{Sicca complaints}

SLE and pSS have overlapping symptomatology and patients with SLE can have secondary Sjögren's syndrome. Therefore, sicca complaints were separately evaluated in order to estimate the potential overlap. Overall, $5.8 \%$ had 
Table 3 Distribution of SLE-CSQ scores among the population-based cohort after exclusion of SLE and other CTDs

\begin{tabular}{crc}
\hline SLE-CSQ score & \multicolumn{1}{l}{$\mathbf{n}$} & Percentage \\
\hline 0 & 41781 & 49.1 \\
1 & 24994 & 29.3 \\
2 & 11719 & 13.8 \\
3 & 4464 & 5.2 \\
4 & 1563 & 1.8 \\
5 & 480 & 0.6 \\
6 & 121 & 0.1 \\
7 & 35 & $<0.01$ \\
8 & 10 & $<0.01$ \\
9 & 2 & $<0.01$ \\
10 & 1 & $<0.01$ \\
11 & 0 & 0 \\
$\geq 4$ & 2212 & 2.6 \\
\hline
\end{tabular}

CTD, connective tissue disease; SLE-CSQ, Connective Tissue Disease Screening Questionnaire focusing on SLE.

both sicca complaints of the eyes and mouth. The incidence of sicca complaints per SLE-CSQ group is shown in online supplemental table 1. With increase of SLE-CSQ score, simultaneously the proportion of participants with sicca complaints increased. Regarding the group with a score $\geq 4,24.2 \%$ had sicca complaints of both eyes and mouth compared with $3.1 \%$ in the group with a score of 0 . Patients with sicca complaints of both eyes and mouth were more often women and more frequently smokers than those without sicca complaints. The frequency of detectable CTD antibodies was not different for individuals with sicca complaints (data not shown).

\section{DISCUSSION}

This study investigated the prevalence of SLE-specific complaints formulated on the CTD-CSQ in the general population. The original CSQ including SLE-specific questions (SLE-CSQ), was developed as the first diagnostic step for a specific population with suspected rheumatological disease. However, it is known that lupus symptoms can be present many years before referral to a rheumatologist, and in hindsight patients with SLE often have visited general practitioners before the referral to a rheumatologist. ${ }^{2} 314$ Majority of the patients have experienced fatigue, joint pain, fever, photosensitivity and myalgia in the year before diagnosis. Therefore, it is of interest to test the prevalence of SLE-specific complaints in the general population, by using the SLE-SCQ, in relation to other characteristics including biochemical values.

Based on the ACR criteria for SLE, answering four or more questions on the SLE-CSQ confirmatively was regarded as an increased suspicion for SLE. ${ }^{8}$ In this northern European population $2.6 \%$ of the participants have $\geq 4$ SLE-related complaints. These individuals were-like patients with SLE-younger and more frequently female than those with none or less positive answers. After correction for age, gender, BMI and smoking status as potential confounders, haemoglobin levels were lower in the group with SLE-CSQ $\geq 4$, whereas neutrophil counts were higher. In accordance, haematological disorders are reported in the literature to be present in $53.5 \%$ of patients with newly diagnosed SLE. ${ }^{3}$ However, increased neutrophils are not typical of SLE.

It has been stipulated that oestrogens might increase the risk of developing SLE. ${ }^{15}$ In the current study, more women in the highest SLE-CSQ scores had ever used OCP. In contrary, the current use of OCP was lower in this group. However, probably the cumulative dose or

Table 4 Percentages of positively answered questions for all (SLE and other CTDs excluded) participants, specified per subgroup based on the total number of positive answers, and patients with SLE and CTD

\begin{tabular}{|c|c|c|c|c|c|c|}
\hline Question & $\begin{array}{l}\text { All } \\
n=85169\end{array}$ & $\begin{array}{l}1 \\
n=24994\end{array}$ & $\begin{array}{l}2-3 \\
n=16183\end{array}$ & $\begin{array}{l}\geq 4 \\
\mathrm{n}=2212\end{array}$ & $\begin{array}{l}\text { SLE } \\
n=46\end{array}$ & $\begin{array}{l}\text { Other CTDs } \\
\mathbf{n}=\mathbf{8 0}\end{array}$ \\
\hline 1. Joint swelling & 4.9 & 6.1 & 12.1 & 32.0 & 28.3 & 30.0 \\
\hline 2. Finger discolouration & 5.7 & 5.9 & 15.4 & 39.6 & 41.3 & 45.0 \\
\hline 3. Ulcers & 6.3 & 6.5 & 16.8 & 45.2 & 41.3 & 25.0 \\
\hline 4. Red rash on cheeks & 2.1 & 1.6 & 5.8 & 19.5 & 41.3 & 12.5 \\
\hline 5. Photosensitivity & 24.1 & 34.5 & 62.4 & 80.1 & 69.6 & 45.0 \\
\hline 6. Pleurisy & 5.6 & 5.0 & 15.4 & 46.1 & 34.8 & 21.3 \\
\hline 7. Alopecia & 6.5 & 5.0 & 19.9 & 50.0 & 34.8 & 15.0 \\
\hline 8. Haematological features & 22.1 & 29.6 & 59.5 & 78.1 & 65.2 & 41.3 \\
\hline 9. Proteinuria & 6.5 & 5.7 & 19.4 & 44.5 & 41.3 & 17.5 \\
\hline 10. Skin lupus & 0.1 & 0 & 0.3 & 2.6 & 67.4 & 12.5 \\
\hline 11. Positive lupus test & 0.1 & 0 & 0.2 & 2.0 & 93.5 & 10.0 \\
\hline
\end{tabular}

CTD, connective tissue disease. 
Table 5 Characteristics in the different groups based on the number of positive SLE-SCQ answers

\begin{tabular}{|c|c|c|c|c|c|}
\hline Group number & 1 & 2 & 3 & 4 & \multirow[b]{3}{*}{$P$ value } \\
\hline SLE-CSQ score & 0 & 1 & $2-3$ & $\geq 4$ & \\
\hline Characteristics & & & & & \\
\hline Gender (female) & 41.9 & 69.3 & 86.0 & 93.0 & $<0.0001$ \\
\hline $\mathrm{BMI}\left(\mathrm{kg} / \mathrm{m}^{2}\right)$ & 25.7 & 25.1 & 24.8 & 24.8 & $<0.0001$ \\
\hline Current (yes) & 18.4 & 19.0 & 18.8 & 20.5 & 0.04 \\
\hline Never (yes) & 49.6 & 48.9 & 48.5 & 47.3 & 0.03 \\
\hline \multicolumn{6}{|l|}{ Women only } \\
\hline Nulliparity (yes) & 17.7 & 19.4 & 17.9 & 18.7 & 0.001 \\
\hline Menopausal (yes) & 8.7 & 6.9 & 6.7 & 6.4 & $<0.0001$ \\
\hline Ever (yes) & 89.5 & 91.1 & 93.7 & 93.4 & $<0.0001$ \\
\hline \multicolumn{6}{|l|}{ Blood levels } \\
\hline Haemoglobin (g/L) & 14.5 & 13.9 & 13.5 & 13.9 & $<0.0001$ \\
\hline Leucocytes $\left(\times 10^{9} / \mathrm{L}\right)$ & 5.7 & 5.8 & 5.7 & 5.9 & 0.004 \\
\hline Lymphocytes (×109/L) & 1.91 & 1.93 & 1.93 & 1.94 & 0.08 \\
\hline Neutrophils $\left(\times 10^{9} / \mathrm{L}\right)$ & 3.1 & 3.1 & 3.1 & 3.2 & $<0.0001$ \\
\hline Monocytes $\left(\times 10^{9} / \mathrm{L}\right)$ & 0.46 & 0.45 & 0.44 & 0.44 & $<0.0001$ \\
\hline Thrombocytes $\left(\times 10^{9} / \mathrm{L}\right)$ & 239 & 249 & 255 & 258 & $<0.0001$ \\
\hline Creatinine $(\mu \mathrm{mol} / \mathrm{L})$ & 76 & 71 & 68 & 67 & $<0.0001$ \\
\hline
\end{tabular}

The outcomes in bold appoint the values that are significantly different from group $4(p<0.05)$.

Data are presented as medians for continuous variables and as percentages for categorical variables.

Kruskal-Wallis analysis was performed for comparison of the four groups. Mann-Whitney was used to compare two groups.

$P$ values $\leq 0.05$ are considered significant.

${ }^{*}$ CTD screen was tested in $25 \%$ of the whole group.

†This percentage refers to the group of CTD screen-positive participants.

$\mathrm{BMI}$, body mass index; CTD, connective tissue disease; OPC, oral conceptive pills; SLE-CSQ, Connective Tissue Disease Screening

Questionnaire focusing on SLE.

duration of OCP use is higher and these data are needed in order to correctly interpret the results.

Among individuals with a reported established diagnosis of SLE, 73.9\% had at least four positive SLE-CSQ questions, reflecting good sensitivity of this questionnaire as demonstrated before. ${ }^{12}$ The fact that sensitivity is not as high as the $96 \%$ that was reported previously could be explained by the fact that patients in the current study did not have newly diagnosed disease. Sicca complaints were tested to check for potential overlap with pSS. Of the participants with a high-risk profile based on SLECSQ, $24.2 \%$ reported sicca complaints of the mouth as well as the eyes. This is comparable with the known prevalence of secondary Sjögren's syndrome in SLE, as it was reported to be $23 \% .^{16}$

Notably, participants with $\geq 4$ positive answers on the SLE-CSQ more frequently expressed CTD-related autoantibodies than the group without any positive answer. The CTD screen detects antibodies against dsDNA and against specific extractable nuclear antibodies (ENA), which are more specific for SLE than the ANA test. Whereas all classifiable patients with SLE express ANA as detected by indirect immunofluorescence, as it is nowadays an entrance criterion for SLE, ${ }^{11}$ not all patients with SLE do express these more specific anti-dsDNA or anti-ENA. Of note, not all individuals with anti-ENA and anti-dsDNA will develop autoimmune diseases. ${ }^{17}$ However, participants in this longitudinal cohort with both high SLE-CSQ scores and positive CTD screen are still at particular interest for further research as they might have the highest risk to progress to SLE.

Whether the SLE-CSQ is of predictive value cannot be interpreted from our results. Therefore, long-term follow-up of this cohort is needed. Young $e t a l^{18}$ followed 
Epidemiology and outcomes

Table 6 Multivariate multinomial logistic analysis, adjusted for age, gender, BMI and smoking status, comparing the different groups based on SLE-CSQ score

\begin{tabular}{|c|c|c|c|c|}
\hline Variable & SLE-CSQ score & OR & 95\% interval & $P$ value \\
\hline \multirow[t]{4}{*}{ Haemoglobin (g/L) } & 0 & 1.58 & 1.47 to 1.72 & $<0.0001$ \\
\hline & 1 & 1.20 & 1.10 to 1.31 & $<0.0001$ \\
\hline & $2-3$ & 1.06 & 0.97 to 1.16 & 0.19 \\
\hline & $\geq 4$ & Ref & & \\
\hline \multirow[t]{4}{*}{ Leucocytes $\left(\times 10^{9} / L\right)$, log-transformed } & 0 & 0.72 & 0.45 to 1.15 & 0.17 \\
\hline & 1 & 0.66 & 0.41 to 1.07 & 0.09 \\
\hline & $2-3$ & 0.51 & 0.32 to 0.84 & 0.007 \\
\hline & $\geq 4$ & Ref & & \\
\hline \multirow[t]{4}{*}{ Neutrophils ( $\left.\times 10^{9} / L\right)$, log-transformed } & 0 & 0.69 & 0.48 to 0.98 & 0.04 \\
\hline & 1 & 0.65 & 0.46 to 0.93 & 0.02 \\
\hline & $2-3$ & 0.56 & 0.39 to 0.80 & 0.002 \\
\hline & $\geq 4$ & Ref & & \\
\hline \multirow[t]{4}{*}{ Monocytes $\left(\times 10^{9} / L\right)$, log-transformed } & 0 & 0.83 & 0.55 to 1.25 & 0.38 \\
\hline & 1 & 0.82 & 0.55 to 1.24 & 0.35 \\
\hline & $2-3$ & 0.71 & 0.47 to 1.07 & 0.10 \\
\hline & $\geq 4$ & Ref & & \\
\hline \multirow[t]{4}{*}{ Thrombocytes $\left(\times 10^{9} / \mathrm{L}\right)$, log-transformed } & 0 & 0.85 & 0.49 to 1.47 & 0.56 \\
\hline & 1 & 0.95 & 0.55 to 1.65 & 0.86 \\
\hline & $2-3$ & 1.03 & 0.59 to 1.80 & 0.93 \\
\hline & $\geq 4$ & Ref & & \\
\hline \multirow[t]{4}{*}{ Creatinine (nmol/L), square root-transformed } & 0 & 1.04 & 0.95 to 1.14 & 0.35 \\
\hline & 1 & 1.04 & 0.95 to 1.14 & 0.39 \\
\hline & $2-3$ & 0.97 & 0.91 to 1.09 & 0.97 \\
\hline & $\geq 4$ & Ref & & \\
\hline \multirow[t]{4}{*}{ CTD screen-positive } & 0 & 0.77 & 0.48 to 1.25 & 0.29 \\
\hline & 1 & 0.95 & 0.61 to 1.59 & 0.95 \\
\hline & $2-3$ & 0.70 & 0.56 to 1.49 & 0.70 \\
\hline & $\geq 4$ & Ref & & \\
\hline \multicolumn{5}{|l|}{ Women only } \\
\hline \multirow[t]{4}{*}{ Nulliparous (yes) } & 0 & 0.75 & 0.65 to 0.87 & $<0.001$ \\
\hline & 1 & 0.80 & 0.80 to 0.69 & 0.003 \\
\hline & $2-3$ & 1.00 & 0.86 to 1.15 & 0.96 \\
\hline & $\geq 4$ & Ref & & \\
\hline \multirow[t]{4}{*}{ Menopausal (yes) } & 0 & 1.31 & 1.07 to 1.59 & 0.009 \\
\hline & 1 & 1.01 & 0.87 to 1.31 & 0.52 \\
\hline & $2-3$ & 1.02 & 0.83 to 1.25 & 0.88 \\
\hline & $\geq 4$ & Ref & & \\
\hline \multirow[t]{4}{*}{ Current OCP use (yes) } & 0 & 1.26 & 1.12 to 1.42 & $<0.001$ \\
\hline & 1 & 1.24 & 1.10 to 1.14 & $<0.001$ \\
\hline & $2-3$ & 1.13 & 1.00 to 1.27 & 0.04 \\
\hline & $\geq 4$ & Ref & & \\
\hline
\end{tabular}

Continued 


\begin{tabular}{lclll}
\hline Table 6 Continued & & & & \\
\hline Variable & SLE-CSQ score & OR & 95\% interval & P value \\
\hline OCP use, ever (yes) & 0 & 0.66 & 0.55 to 0.80 & $<0.001$ \\
& 1 & 0.83 & 0.69 to 1.00 & 0.05 \\
& $2-3$ & 1.09 & 0.90 to 1.31 & 0.40
\end{tabular}

$P$ values $\leq 0.05$ are considered significant.

BMI, body mass index; CTD, connective tissue disease; OCP, oral anticonceptive pills; Ref, reference; SLE-CSQ, Connective Tissue Disease Screening Questionnaire focusing on SLE.

a group of 364 family members of patients with SLE for a mean of 6.3 years. Of the 22 participants who transitioned to classified SLE, 16 (73\%) had an SLE-CSQ score of $\geq 4$ compared with 72 of 353 (20\%) individuals who did not develop SLE. In family members with a score $\geq 3$, the positive predictive value for developing SLE was $15 \%$ and the negative predictive value was $99 \%$ in the study referred to.

It would be interesting to further test the SLE-CSQ as a screening instrument in the population. This questionnaire could be used in first-line medical care, preferably as part of a stepwise design. The next step would then be to test ANA in individuals with high suspicion for SLE based on the questionnaire. Those individuals with a positive ANA could be referred to a medical specialist for further assessment and follow-up to evaluate which percentage develop a CTD.

Although this is a large population cohort, there are some limitations. Most importantly, the diagnosis of SLE and CTD was self-reported by participants. There was no access to medical files, so it could not be checked if the classification criteria were met, although the use of immunosuppressive medication was confirmed. Second, the population is mainly Caucasian, while the prevalence of SLE is higher in other ethnicities. Therefore, it would be interesting to repeat this research in a more diverse population. One other limitation is the fact that blood samples were not retrieved at the same time as the questionnaire. The median time between these testing moments was 25 months. It is possible that in the mean time CTD autoantibodies have developed or other blood tests have changed. Not all but 85295 (51\%) of 167729 subjects have filled in the follow-up questionnaire, which could have resulted in a selection bias. Unfortunately, ANA was not tested in this population cohort. As ANA is more prevalent and more commonly tested than anti-dsDNA and anti-ENA, it would have been of interest. The SLE-CSQ score used in this study was retrieved from but not exactly the same as in the original publication. Hence the validity and applicability may have been slightly changed. Lastly, patients with rheumatoid arthritis (RA) have not been excluded from this study. Potentially, as RA is a systemic disease, these patients could have higher SLE-CSQ scores, which could influence the data. However, a specificity of $85 \%$ for SLE has been reported for this questionnaire.

In conclusion, the prevalence of $\geq 4$ SLE-related symptoms as tested by the SLE-CSQ is $2.6 \%$ in the northern
Dutch population. These individuals are younger and have a female predominance $(90 \%)$. Persons suspicious for SLE based on this questionnaire have, when corrected for potential confounders, higher neutrophil counts and lower levels of haemoglobin. This study supports the usefulness of further research on screening for SLE in the general population by providing insight into the prevalence of SLE symptoms. This might help in developing a stepwise approach in order to diagnose SLE earlier. However, longer follow-up of this cohort is necessary to show the predictive value of the SLE-CSQ score in combination with other promising variables like autoantibodies in a population-based cohort.

Contributors All authors made substantial contributions to either the conception of the work, or the acquisition, analysis or interpretation of data. WL collected, analysed and interpreted the data, and wrote the first version of the manuscript. SA contributed to the design of the work and analysed the data. CR contributed to acquisition of the laboratory data and drafted the manuscript. EB and HB drafted and substantially revised the manuscript. JW and KdL designed the work and substantively revised the work. The authors agree to be accountable for all aspects of the work in ensuring that questions related to the accuracy or integrity of any part of the work are appropriately investigated and resolved.

Funding This study was supported by the Dutch Arthritis Association and an inkind grant from ThermoFisher Scientific as part of the IMI JU-funded project BeTheCure (contract no 115142-2) and by a grant from Biobanking and Biomolecular Research Infrastructure (BBMRI)-NL complementation projects. The Lifelines Initiative has been made possible by subsidy from the Dutch Ministry of Health, Welfare and Sport, the Dutch Ministry of Economic Affairs, the University Medical Center Groningen (UMCG), Groningen University, and the provinces in the north of the Netherlands (Drenthe, Friesland and Groningen).

Competing interests None declared.

Patient consent for publication Not required.

Ethics approval The Lifelines cohort study was conducted according to the principles of the Declaration of Helsinki and approved by the local ethics committee of the University Medical Center Groningen (UMCG).

Provenance and peer review Not commissioned; externally peer reviewed.

Data availability statement Data may be obtained from a third party and are not publicly available.

Supplemental material This content has been supplied by the author(s). It has not been vetted by BMJ Publishing Group Limited (BMJ) and may not have been peer-reviewed. Any opinions or recommendations discussed are solely those of the author(s) and are not endorsed by BMJ. BMJ disclaims all liability and responsibility arising from any reliance placed on the content. Where the content includes any translated material, BMJ does not warrant the accuracy and reliability of the translations (including but not limited to local regulations, clinical guidelines, terminology, drug names and drug dosages), and is not responsible for any error and/or omissions arising from translation and adaptation or otherwise.

Open access This is an open access article distributed in accordance with the Creative Commons Attribution Non Commercial (CC BY-NC 4.0) license, which permits others to distribute, remix, adapt, build upon this work non-commercially, 
and license their derivative works on different terms, provided the original work is properly cited, appropriate credit is given, any changes made indicated, and the use is non-commercial. See: http://creativecommons.org/licenses/by-nc/4.0/.

\section{ORCID iD}

Wietske Lambers http://orcid.org/0000-0002-2026-5191

\section{REFERENCES}

1 Pons-Estel GJ, Alarcón GS, Scofield L, et al. Understanding the epidemiology and progression of systemic lupus erythematosus. Semin Arthritis Rheum 2010;39:257-68.

2 Rees F, Doherty M, Lanyon P, et al. Early clinical features in systemic lupus erythematosus: can they be used to achieve earlier diagnosis? a risk prediction model. Arthritis Care Res 2017;69:833-41.

3 Sebastiani GD, Prevete I, Iuliano A, et al. Early lupus project: oneyear follow-up of an Italian cohort of patients with systemic lupus erythematosus of recent onset. Lupus 2018;27:1479-88.

4 Arbuckle MR, McClain MT, Rubertone MV, et al. Development of autoantibodies before the clinical onset of systemic lupus erythematosus. N Engl J Med 2003;349:1526-33.

5 Eriksson $\mathrm{C}$, Kokkonen $\mathrm{H}$, Johansson M, et al. Autoantibodies predate the onset of systemic lupus erythematosus in northern Sweden. Arthritis Res Ther 2011;13:R30.

6 Segura BT, Bernstein BS, McDonnell T, et al. Damage accrual and mortality over long-term follow-up in 300 patients with systemic lupus erythematosus in a multi-ethnic British cohort. Rheumatology 2020;59:698-33.

7 James JA, Kim-Howard XR, Bruner BF, et al. Hydroxychloroquine sulfate treatment is associated with later onset of systemic lupus erythematosus. Lupus 2007;16:401-9.
8 Tan EM, Cohen AS, Fries JF, et al. The 1982 revised criteria for the classification of systemic lupus erythematosus. Arthritis Rheum 1982;25:1271-7.

9 Hochberg MC. Updating the American College of rheumatology revised criteria for the classification of systemic lupus erythematosus. Arthritis \& Rheumatism 1997;40:1725-34.

10 Petri M, Orbai A-M, Alarcón GS, et al. Derivation and validation of the systemic lupus international collaborating clinics classification criteria for systemic lupus erythematosus. Arthritis Rheum 2012;64:2677-86.

11 Aringer M, Costenbader K, Daikh D, et al. 2019 European League against Rheumatism/American College of rheumatology classification criteria for systemic lupus erythematosus. Ann Rheum Dis 2019;78;::1151-9. ;0.

12 Karlson EW, Sanchez-Guerrero J, Wright EA, et al. A connective tissue disease screening questionnaire for population studies. Ann Epidemiol 1995;5:297-302.

13 Munroe ME, Young KA, Kamen DL, et al. Discerning risk of disease transition in relatives of systemic lupus erythematosus patients utilizing soluble mediators and clinical features. Arthritis Rheumatol 2017;69:630-42.

14 Leuchten N, Milke B, Winkler-Rohlfing B, et al. Early symptoms of systemic lupus erythematosus (SLE) recalled by 339 SLE patients. Lupus 2018;27:1431-6.

15 Moulton VR. Sex hormones in acquired immunity and autoimmune disease. Front Immunol 2018;9:1-21.

16 Ruacho G, Kvarnström M, Zickert A, et al. Sjögren syndrome in systemic lupus erythematosus: a subset characterized by a systemic inflammatory state. J Rheumatol 2020;47:865-75.

17 Didier K, Bolko L, Giusti D, et al. Autoantibodies associated with connective tissue diseases: what meaning for clinicians? Front Immunol 2018;9:541.

18 Young KA, Munroe ME, Guthridge JM, et al. Screening characteristics for enrichment of individuals at higher risk for transitioning to classified SLE. Lupus 2019;28:597-606. 